\title{
Research on Chinese-character Image Streaming based on the Digital-Streaming Media Technology
}

\author{
Lin Liu $^{1, a}$, Jiaxin Wang ${ }^{2, b}$ \\ ${ }^{1}$ School Of Art Design, Hubei University Of Technology, Wuhan, Hubei Province, China \\ ${ }^{2}$ School Of Art Design, Hubei University Of Technology, Wuhan, Hubei Province, China
}

\begin{abstract}
The survival mode of the font design has been changed to the mode of imaging design by the new-media technology. The Chinese character image could achieve different image effects of fonts including flow, page turning, rotation, and scrolling. Streaming media technology achieve the streaming playing approach to discuss the composition and dynamic effect of Chinese character image streaming. Based on the characteristics of digital-streaming media, particle and fluid technology, and Chinese character image streaming, this paper will analyze and sort out the formation and techniques of Chinese character image based on streaming media technology.

This paper proposed the new concept of 'Chinese character image streaming'. Since there is no systematic theories and no summarized experience about the practical works regarding the Chinese character image streaming, this paper compared and analyzed this new concept with above-mentioned technologies to provide new materials for the researches of Chinese character designs in the future.
\end{abstract}

\section{Introduction}

Multimedia design is expressing mode integrating text, sound, image, graphics, animation, and video to show intuitive, vivid, life-like image and sound simultaneously, where light effect and motion are the important factors to show the visual characteristics of Chinese characters. Through the transmissions of the digital-streaming media, the real-time playing of animations, videos and music in the internet could be achieved, which means that Chinese character images are played using streaming technology with the characteristics of the streaming. The Chinese character image streaming contains the means of streaming media. Meanwhile, 3D-flowing fonts are achieved by the special effect of particle and fluid, which are the main elements to form the Chinese character image streaming, while the art of Chinese character image streaming could not be achieved without the special effects. The particle and fluid special technology in CG special effect technology assist the development of Chinese character image to be shown as flowing mode for watching. The cross-use of particle and fluid technology could make combination of word shapes of burst and flow, such as broken font, font forming with flame, and font forming with spray. Those fonts are multi-dimensional, dynamic, and flowing, which could achieve dynamic effect of the opening texts.

\section{Steaming Media and Particle and Fluid Technology}

\subsection{Heading Level 2 Transmission Technology of Streaming Media}

The development of network, communication and multimedia technology has significant effect on the internet. Based on the limited bandwidth and crowded dial-up network, the best solution to the transmission of video, music, and animation in the narrow-band network is to use the transmission of streaming media. The concept of steaming is not a new one, it has been used in the radio and television for a long time. There is no storage to signals of the traditional radio and television, which are transmitted from the transmitter to the receiver in real time without any delay. In the past, multi-media files could be played after downloading from the server ${ }^{1}$. Taking a one-minute-small video as an example, the downloading time was more than 30 minutes in the $56 \mathrm{~K}$ narrow-band internet, which is a significant limitation to the interaction by using substantial video information in the internet.

With the development of the internet speed and audio and video compression technology, the streaming transmission is used to real-timely transmit video media or multi-media information fluently to use the streaming technology in the IP internet. As a result, users could play and watch the streaming-media files such as animations and videos uncompressed by the corresponding player or 
other hardware and software in the users' computer in few seconds or dozens of seconds of start-up delay, instead of downing the whole file to watch the file content by the non-streaming play, and the rest part of the file will be continuously downloaded at the background server ${ }^{2}$. Streaming technology will create a buffer on client's computer to store a piece of data in advance. When the actual network speed is smaller than the speed of playing, the player will use this part of data to avoid the interruption and quality of playing. Some people vividly compare the streaming media to a shower. The body is been washing while water is flowing, instead of washing after the bathtub is full of water. There are two streaming transmissions: progressive streaming transmission and real-time streaming transmission.

Progressive streaming transmission (PST) is the sequential download, users could watch the online media while downloading, but they could not watch the parts which have not been underloaded. PST could not adjust the speed automatically. Since the standard HTTP server could transmit this type of files, it is also named as HTTP streaming transmission. PST is more suitable to transmit high-quality short clips, such as the opening, ending, and advertisement. The final playing quality is ensured by the non-lose downloading of the file before it has been watched. PST store files in the standard HTTP or FTP server, which is convenient to be managed, and basically independent from the firewall.

Real-time streaming transmission (RST) is different from the HTTP streaming transmission, which requires special media server and transmission conditions. RST generally transmits information in real-time mode, such as on-site events and live broadcast. In theory, the playing of the real-time streaming could not be stopped, if the speed of the narrow-band internet of users is not enough or there are some problems in the net, the playing will be paused to lose real-time information with poor video quality. In general, The RST is suitable for broadcast media rather than image transmission.

RST generally need special servers and players. In the implementation of streaming transmission, the RTP / UDP and RTSP / TCP are used to establish the connection with $\mathrm{A} / \mathrm{V}$ server to redirect the output of the server to a destination address different from that running the A/V Helper program in the users' computer. The fundamental principle is given in Figure 1.

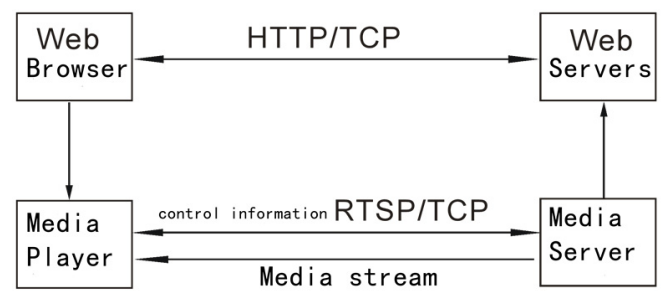

Figure 1: The transmission of streaming media ${ }^{6}$

Digital streaming medias contain the streaming of audio, video, text, image and animation. Steaming-media technology could achieve the broadcast or combination of multiple videos, images, and text files to be played in the same screen, achieve the effects of page turning, rotation, or image blur, and achieve dynamic or scrolling effect of the titles. By using streaming-media editing software with video capture card and non-linear editing software, the editing of streaming-media file could be completed. If we watch the video clips of the Spring Festival Evening Show, this is only the technology of watching, where the $\mathrm{CG}$ special-effect technology is essential to the achievement of the special effect of image-video art.

\subsection{Particle and Fluid Technology}

Particle and fluid are the two common techniques in the $\mathrm{CG}$ special effects. The transfiguring works of text deduction, flowing fluid, and even the ink effects are inseparable from the fluid technology. Generally speaking, the fluid technology is developed based on the particles, in which thousands of particles form the final effect of fluid by the meshing method ${ }^{4}$. The simulation of particle animations is easier and more controllable than the fluid animation in the theory, since fluid is responsible for the volume rather than the dynamic effect. However, in the actual producing process, there is a relationship between the dynamic motion and material properties. Reliable animation effect could be achieved by the combination of particle and fluid technology.

Particle system is a technology mentioned by Reeves to simulate the natural scenes in $1983^{5}$. The definition of particles is the smallest substance existing in free motion, and particles generally gather vitality and energy in the state of motion rather than remaining the relatively static state. Each particle has its own life cycle and has to go through the three stages of "generation", "move and growth" and "death" in the system. Therefore, unlike traditional methods that can only generate instantaneous and static scenes, a series of motion evolution images can be generated, which makes simulating dynamic natural scenes the first choice for particle special effects. The dynamic particle technology in Maya software platform, simulations and animations are used to achieve the explosion, whirlwind and starry sky. The dynamic particle system in Maya is very advanced, since it allows relative less use of motions to control particles and the cross-use of different animation tools ${ }^{6}$. There are some interactions among particles, including attraction, mutual repulsion, and generations of new particles. The Galaxy and the explosion scene are the typical states of particles shown in Figure 2. Due to the free motion of particles itself, it could be formed to various animation effects to be played, including divergence from a point, free diffusion or from repeat focus and fusion state to from a burst shape.

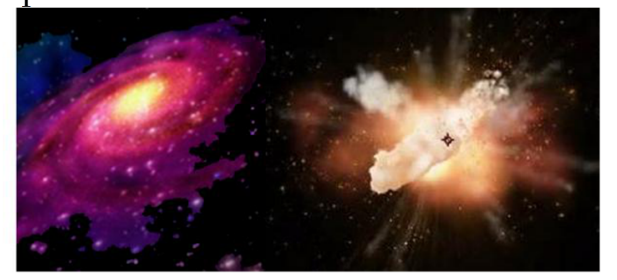

Figure 2: The Galaxy and explosion by particle technology 
The particle technology and fluid technology are different, since the particle is calculated with the single point in the space, which means that every particle moves independently to other particles where the viscous fluid simulation is difficult to be applied to particles. Fluid is the object that will occur continuous deformation by the force on the unit area. There is no certain shape of fluid, which could be changed to any shape or split. Gas and liquid are fluid 7 , including river, rain, and cloud. However, particle could actually be affected by the fluid in the container to form a component in the formation of fluid effect.

The fluid special effect is generally to set properties with image attachment to collide with other geometric objects, influence soft bodies, and interact with particles. The principle of the fluid effect is when the characteristics of fluid are set as fluid grid, the attribute values will be resolved, which is that every attribute value could control the fluid to perform vivid simulation presentation. Voxel is the essentially operational concept in the fluid grid, the resolving tool will calculate the values in the grids, where the changes of values will develop the representation of fluid ${ }^{8}$. Fluid animation has special Realflow software to calculate the precise motion trajectory by the connection between pug-ins and imagemaking software, and particles to simulate flowing and collision of the liquid and gas, and use the smooth polygon meshed by particles to simulate the motion speed and produce the special effects of splash, wave, ink, flame and smoke.

Based on the basic description to particle and fluid technology, since particle animation is easier to be controlled than fluid animation simulation based on physic, it is widely used and combined with the volume, property, and performance of fluid technology to achieve realistic performance and dynamic effect, including broken font, font forming with flame, and font forming with spray, and the fonts are multi-dimensional, dynamic, and flowing.

\section{Features of Chinese character Image Streaming}

The so-called Image stream of Chinese characters is based on the digital stream, or "streaming media" image mode of Chinese characters. The Chinese character design consists of images and transmits information in a "stream" mode through a video screen. "stream" has a way to watch streaming media here, and also has the meaning of image stream. The $3 \mathrm{~d}$ animated fonts realized by particle effect technology and the 3D flowing fonts realized by fluid effect constitute the basic features of image flow.

\subsection{Animation Font of Chinese Character}

The animation font of Chinese character refers to 3D dynamic Chinese character image designed and produced by $3 \mathrm{D}$ animation software, which could be continuously viewed. The $3 \mathrm{D}$ opening text with special dynamic motion could be produced by $\mathrm{CG}$ particle and fluid special-effect technology. An illustration is the transformation of animation font, which means that one font is developed to another font by the evolution process. The special effect of the falling font in the opening of 'Total Soccer' of CCTV5 is to use the unique deformation feature of particles to perform the explosion effect of the animation font. This case has used the explosive effect in the particle special effect, and the font can have the performances of flowing, rotation, scrolling, and jumping. In the post-production of light effect in the opening of 'Case Tracing', dynamic characters (rotation, jumping and flipping) are accompanied by the changes of the camera shooting, which means the dynamic effect could be enhanced by the light-effect post-production.

Hunan satellite TV "day day up" opening, the actual filming and CG special effects technology, opening around the columns with text, with a pencil and ruler is out of the "day day up" text outline draft, from handdrawn signs sunflower design diagram to stone carving "day day up" symbol modelling to use 3D dynamic text from global to local rendering effect; From the fixed plate to the title text, sunflower patterns, the combination of human figures into the image. Column around the theme "Day Day up" the formation process of the font design and the rotation of the brothers appear alternately, showing that each different frame based on the time change of the screen in the mind of the audience produces the sense of "movement". Column screen text and sunflower pattern combination logo, the stroke of "upward" is designed as an upward arrow, which reflects the health, upward, positive, humorous, connotation.

\subsection{Flowing font of Chinese Character}

Flowing font is based on two dimensions. The first one is presented on the LED screen, which is a flowing font that displayed on the electronic billboard. When the advertisement is playing, fonts are coming from the right edge of the screen and flowing to the left edge, and the fonts will disappear when flowing out of the left edge. The second one is short video clip formed by twodimension characters, which is a short clip of characters with narrative plotting. It contains the size change of the characters, combination of characters and graphics, different fonts of characters to form the image. The third one is the video opening made by ink special-effect technology, which is the three-dimension dynamic flowing fonts developed from the combination of real shooting, calligraphy, traditional Chinese painting, and brushwork. The two-dimension flowing font design displayed on the screen generally could be achieved by the use of Photoshop, Flash, After effect. It is also applied to the video of meeting information in the public area or advertisement presentation or product publicity, and the principle of design and production of this technology is relatively easy and simple, which would not be mentioned in this paper.

From the literal point of view, flowing font is a font that is displayed by flowing method on the screen, which do not cover the art of the font. Comparing with animation font, flowing font emphasises the effect of 
flowing, which is that different story clips are narrated by the font flowing. Hence, the font is an important factor to narrate stories and transmit information. The implementation of expressing form of advertisement video, short video clip, games based on web page, and publication of on-screen information based on the flowing font of Chinese characters is getting significantly popular. Based on an event as the plot, the video is produced by the combination of language and flowing font, and the rate change of fast or slow of the font is combined with language audio, fonts in different styles, scenes, and the ending of characters to form the image.

The method of combining two-dimension and threedimension image formed with characters and image, and calligraphy with ink special effect to produce flowing font in the opening is frequently used. The first reason is that, it could take the experience from motion-captured technology and camera-path-matching technology. In a real-shooting motion video, the tracked time period and image area are selected, the software then automatically process the numerical change of the motion path in accompanied with the time change, and it could be developed as three-dimension image element. Secondly, frog-liking effect could be firstly developed in the fluid technology in the three-dimension software, and it will then be adjusted to link special effect. Thirdly, it could be achieved in the relative functions in some postproduction software. The Particular particle pug-in in the Trapcode of After Effects software contains the special effect of fire and smoke in the pre-set animation to be used to present the form of ink.

Ink special effect is a displaying form of ink drop, ink line and ink mark of the calligraphy. To achieve this special effect, the key is to produce the irregular effect of the edge fracture and corrosion. The flowing-font characteristics of Chinese character is represented by the flowing effect producing from these irregular edge fracture and corrosion.

For example, BTV science and education channel's design for the beginning of "ink and wash theme chapter"- "wisdom". The opening of the film uses the method of special effects of ink and wash, with the flow track of ink and wash to express the two characters of "heaven and earth" of the torrential cyclone, smooth, rigid and soft, just like the spirit of ethereal, with the momentum of flying in the air, the font is round and vigorous, unrestrained and free. Based on animation flow font and font composition of Chinese character image, is based on flow dynamic or picture forms, changing Chinese character images, like a picture of an image to the people flow changes, both the graphic transformation, and insert text, text converted into images, text is accompanied by music tempo and produce change, create a new experience of visual motion.

\section{Conclusion}

Through the dissemination of streaming media, real-time playback of multimedia such as animation and video on the Internet is realized, that is, the Chinese character images are played in a "streaming" way as media, which has the characteristics of "streaming". The particle and fluid special effect technology in $C G$ technology gave birth to the image of Chinese characters entering our field of vision in "streaming". The intersecting use of particles and fluids can combine words into bursts and flowing shapes. The important reason for multimedia art is the smooth, real, and moving pictures displayed on the screen.

The digital streaming media technology contains the elements of light, color, time and motion, audio, and virtual interaction, and the production of the light and color in the CG special effect is significantly diverse. This industry improves the technology of the post production to the three-dimension opening design, which consists of the common functions of intermediate frame generation and layered synthesis editing. Meanwhile, the key frame technology could continuously achieve space and time illusion from static to dynamic for the opening font or fonts in short video. Streaming media technology further develops multiple effects of the flowing of the Chinese character image by the technology of particle, fluid, and ink. The flowing fonts made from the streaming technology is displayed by the dynamic or flowing image to form the continuous changes of Chinese character. This process continuously displays different images to people with transformation of graphics, text insertion, characters transformed to graphics, characters changed accompanied with the change of the music beat, to create a new experience of visual dynamic sense.

\section{REFERENCES}

1. Li W. 2004, 'Media, Multi-media, Streaming Media and Super Media' $[\mathrm{J}]$, Chinese data and Communication, Vol. 3, Page. 28th.

2. Chen S. 2003, 'Overall Description of the Streaming Media Technology' [J], Journal of Beijing Broadcasting Institute (Science and Technology), Vol. 3, Page. 59th.

3. Wang H. 2010, 'Art of the Digital Media' [M], Huazhong University of Science and Technology Press, Page. 81th.

4. $\mathrm{Xu}$ Q. 2012, 'Secrets of CG Television Transfiguring' [M], People's Post and Telecommunication Press in China, Page. 133th.

5. William T. 1983, 'Particle Systems - A Technique for Modeling a Class of Fuzzy Objects' , Computer Graphics, (SIGGRAPH 83), Vol. 17 (3), PP. 359376.

6. Wang H. 2010, 'Art of the Digital Media' [M], Huazhong University of Science and Technology Press, Page. 248th.

7. Wang Y. 2012, 'Secrets of CG Special Effect Production of Videos' [M], People's Post and Telecommunication Press in China, Page. 216th.

8. Mao H. 2009, 'MAYA Particle and Fluid Special Effect Management' [D], Shanghai Jiao Tong University, Page. 37th. 